\title{
Parent Disciplining Styles to Prevent Children's Misbehaviors toward a Social Robot
}

\author{
Jorge Gallego-Perez \\ ATR Intelligent Robotics and \\ Communication Laboratory \\ 2-2-2 Hikaridai, Seika-cho \\ Keihanna Science City, Kyoto, Japan \\ jgallego@atr.jp
}

\author{
Kazuo Hiraki \\ Dpt. of General Systems Studies Graduate \\ School of Arts and Sciences \\ University of Tokyo \\ 3-8-1, Komaba, Tokyo, Japan \\ hiraki-lab@ardbeg.c.u-tokyo.ac.jp \\ Takayuki Kanda \\ Dpt. of Social Informatics, \\ Kyoto University \\ Yoshida-honmachi, Sakyoku, Kyoto, 606- \\ 8501, Japan \\ kanda@i.kyoto-u.ac.jp
}

\author{
Yasuhiro Kanakogi \\ Dpt. of Psychology \\ Otemon Gakuin University \\ 2-1-15, Nishiai, Ibaraki \\ Osaka, Japan \\ yakanakogi@gmail.com
}

\begin{abstract}
In this paper we present a lab study on robot abuse by children. 61 Japanese children of ages 7-9 interacted individually with Robovie, a social robot, in a context that promoted children's free disruptive behaviors towards the robot. We compared the robot's use of an adaptation of a parental discipline strategy, the so-called love-withdrawal technique, to a similar set of robot behaviors that lacked any specific strategy (neutral condition). The main insight we gained was that perhaps we should better not focus on general robot behaviors to try to fit all children, but rather, we should adapt the robot behaviors to children's individual differences. For instance, we found that the lovewithdrawal-based strategy was significantly more effective in children of age 8-9 than on children of 7 .
\end{abstract}

\section{CCS CONCEPTS}

- Human-centered computing $\rightarrow$ Collaborative and social computing $\rightarrow$ Empirical studies on collaborative and social computing

\section{KEYWORDS}

Human-Robot Interaction, children, robot abuse, parental discipline

\section{ACM Reference format:}

Jorge Gallego-Perez, Kazuo Hiraki, Yasuhiro Kanakogi and Takayuki Kanda. 2018. Parent Disciplining Styles to Prevent Children's Misbehaviors toward a Social Robot. In Proceedings of the 7th International Conference on Human-Agent Interaction (HAI '19). ACM, Kyoto, fapan, 9 pages. https://doi.org/10.1145/3349537.3351903

Permission to make digital or hard copies of all or part of this work for personal or classroom use is granted without fee provided that copies are not made or distributed for profit or commercial advantage and that copies bear this notice and the full citation on the first page. Copyrights for components of this work owned by others than ACM must be honored. Abstracting with credit is permitted. To copy otherwise, or republish, to post on servers or to redistribute to lists, requires prior specific permission and/or a fee. Request permissions from Permissions@acm.org.

HAI '19, October 6-10, 2019, Kyoto, Japan

(C) 2019 Association for Computing Machinery.

ACM ISBN 978-1-4503-6922-0/19/10 \$15.00

https://doi.org/10.1145/3349537.3351903

\section{INTRODUCTION}

From the diverse types of human users that may interact with robots, children prove to be a special population, for example, because children tend to perceive and react to robots in different ways than adults, e.g. children anthropomorphize robots more [1].

However, children are a special population also in terms of selfcontrol and misbehaving. We find a high prevalence of children's misbehaviors for example in animal abuse [2]. The authors in [2] explored among other variables the age of first incident at which participants had committed various forms of animal mistreatment. In the category of hurting/torturing an animal, $11.1 \%$ of participants had perpetrated their first animal abuse when they were 2-6 years old; $50.0 \%$ when they were 6-12 years old; $33.3 \%$ when they were teenagers; and only $5.6 \%$ when they were adults.

In addition to animal mistreatment, children appear also more prone to abuse robots as compared with other age groups (see section 2.2). We could argue that abusing robots is problematic or unethical in itself. But, furthermore, if children have the opportunity to abuse robots and this is not prevented (by adults or by the robots themselves), children may end up enjoying treating them badly. In the long run, this may become a habit and they might develop a natural tendency to mistreat also other people (e.g. [3], [4]).

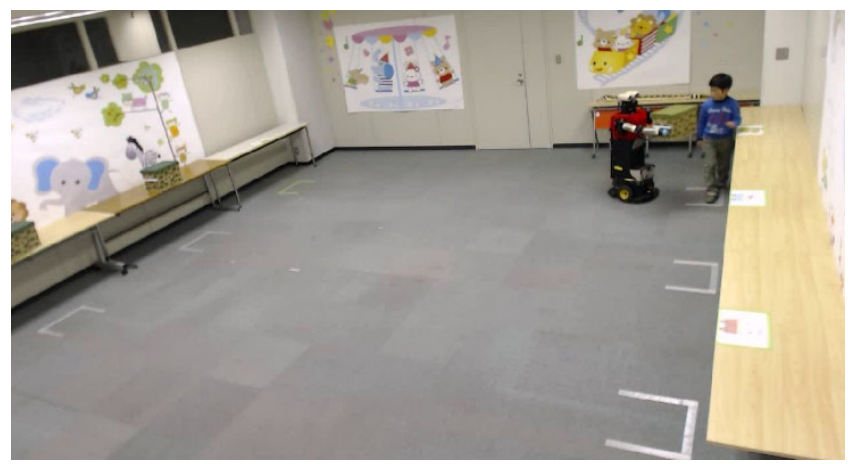

Figure 1: participant blocking the robot's path, disabling it to perform its task. 
Children's frequent lack of discipline may also pose several problems. For example, children sometimes are reluctant to stop bad behaviors when they are asked to do so. As we will see in section 2.3, certain discipline styles of parents are crucial in the raising of children and can help regulate their behavior. From that range of discipline styles, we payed a special attention to Hoffman's parenting strategies [5], which, given their great effectiveness to reduce child misbehavior, we tried to adapt to the context of Human-Robot Interaction. More specifically, we followed Hoffman's love-withdrawal parenting strategy [5] (see section 2.3) and adapted it for a child-robot interaction with the robotic platform Robovie (see Figure 1), with the aim to reduce misbehaviors toward a robot. In light of the above, our research question in this work will be: will an adaptation of the lovewithdrawal strategy be effective at reducing robot abuse?

\section{RELATED WORKS}

To situate the present work in its wider context, in this section we will introduce related works on child Human-Robot Interaction (cHRI), robot abuse, and discipline styles and robot bonding.

\subsection{Child Human-Robot Interaction (cHRI)}

HRI with children presents some peculiarities. Children are not just younger, smaller adults. Their different and immature cognitive development makes them see robots not as mechanical devices but as living systems. It seems that pretend play and anthropomorphization play a relevant role in the ability of children to treat robots as life-like agent [6].

Social robots for children have been employed in a wide variety of contexts, being education and healthcare two of the most notable ones. The use of robots in education for children has been prominent, given that robots may provide cheap, personalized and tireless tutoring, and they often exceed the effectiveness of computer-based tutoring systems [6]. An example is the use of robots to teach English in Japanese schools (e.g. [7], [8]), or to learn about healthy diets and nutrition [9].

In the context of healthcare, robotic pets have proven useful in situations in which animal assisted therapy (AAT) would have been difficult due to hygiene reasons or high costs [6]. Another successful example within the healthcare context has been the use of social robots for children with Autistic Spectrum Disorders (ASD) with robots serving as intermediaries between human therapists and children [10].

Thus, children have interacted with robots in diverse contexts, whereby robots have proven to solve or alleviate a wide range of problems.

\subsection{Robot Abuse}

Few studies have directly addressed the topic of robot abuse (e.g. [11], [12]). For instance, Salvini et al. [11] described how younger people tended to "bully" a social robot at a science fair demonstration in South Korea. The robot was usually approached in groups rather than individually. The "disturbing" behaviors consisted mostly in blocking the robot way, covering the onboard cameras and other sensors with their hands, pushing the bumpers, etc., including also more aggressive behaviors like kicking, punching and slapping the robot [11].

Brščić et al. were the first authors whose explicit aim was to minimize the robot abuse occasioned by children [12]. They carried out a study in which Robovie (Figures 1-4), roamed around in a popular shopping mall in Osaka, Japan. They found a high prevalence of attacks and misbehaviors of children toward the robot, including mainly path obstruction, but also offensive utterances and violence [12], quite in the same vein as what Salvini et al. had found before [11]. They also noticed that this type of misconduct was always performed by children; adults were never involved [12]. A strategy of having the robot react to the children's misbehaviors through verbal expressions, such as "I wish to go through, could you please open the way?", did not prove effective, not even when the robot's wording adopted stronger, emotional or polite expressions.

The authors created a model to estimate the optimum trajectories the robot should follow to minimize the abuse from children, which resulted in having the robot moving in a straight line at full speed when there were no children around, as well as moving to areas with more density of people, or parents, whenever children made their appearance. Although effective, such avoidance behavior would not prove useful in situations in which a robot is unable to physically escape. Thus, the question still remained as to how to design effective robot behaviors that would minimize abuse by children.

\subsection{Discipline Styles and Robot Bonding}

Our work is novel for the fact that we adapted strategies from parenting strategies, which are connected to child rearing practices. To be more precise, we focused on the parenting strategies from Hoffman [5], who presented three parent discipline styles: power assertion, induction and lovewithdrawal. Power assertion refers to applying direct or indirect force over the child or his/her environment (i.e. punishment); induction involves letting the child know how his/her actions may lead to harm other people; and love-withdrawal expresses parents' disapproval by removing emotional support (in a sense, emotional blackmail, such as in the statement "I'm ashamed to be your mother") [5].

We enunciated earlier our research question: will a lovewithdrawal strategy adaptation be effective at reducing robot abuse?). Thus, we included the love-withdrawal strategy in the set of robot behaviors in this study, thereby becoming the first, to our knowledge, who explicitly adapted Hoffman's strategies to research in HRI. We hoped that the usefulness of this strategy in the parent-to-child interaction would generalize to interactions with a robot.

Building a bond with a parent, or robot in our case, is necessary for the love-withdrawal strategy to work, thus, we also included a series of robot bonding strategies. One such technique is selfdisclosure, by which a robot tells the participant information about itself (e.g. a secret). Kanda et al. [13], for instance, had a guide robot in a shopping mall express "personal preferences" like its favorite food, or reveal the fact that it was its second day working there [13]. Also, numerous HRI authors have payed close attention to the anthropomorphism of the robot (e.g. [14]), the intrinsic motivation of the user when interacting with a robot (e.g. [15]), robot gaze (e.g. [16]), etc.

These are some of the strategies that we implemented to increase the bond between child and robot. 


\section{METHODS}

In this section we will outline the experimental design, as well as describe the participants sample, the robot platform we used and the environment in which we conducted the experiment. Subsequently and in timely order we will explain the several phases of the procedure. Next, we will describe the differences between the two experimental conditions. Finally, we will conclude with the data collection and measures.

\subsection{Experimental Design}

A main focus of our experimental design was to allow the participants to behave as spontaneously as possible, creating a situation where children might freely choose to misbehave toward the robot, thereby needing to be disciplined. Thus, they were left with a robot, without attendance from adults and with no specific instructions about what to do in this situation; we tried to give them the impression that staying in the room with the robot was incidental (they just needed to wait until their parent picked them up). To make them believe that they were not participating in an experiment, they just had a brief interaction with the robot and which we referred to as "the experiment" (when it was a fake experiment in reality).

The fact that children received no explicit instructions about how to interact with the robot may be methodologically more disadvantageous than a scripted scenario on one hand (i.e. we had less control, and also there were many participants that would not interact at all with the robot), but on the other hand it should improve the internal validity.

Thus, participants may or may not show forms of misbehavior towards the robot. They all went through the same experimental procedure, and the data from those who showed misbehaviors were analyzed.

\subsection{Participants}

Participants were recruited via an external party that recruited children that lived in the area. At every point in time, we tried to keep constant across conditions the number of participants that misbehaved at least once towards the robot (i.e. participants whose data we would later analyze). Thus, when a new participant arrived, this would be assigned to the condition that had a lowest number of participants that had misbehaved, or would be randomly assigned to one of the two conditions if such ratio was equal across conditions. In total, 61 valid participants were selected for posterior data analysis (20 female, 41 male; children of age 7-8: 15 , children of age 9: 46 , Mean $=8.66, \mathrm{SD}=$ 0.66). Throughout the experiment we found important differences between these two age groups. We attributed these differences to the fact that children of age 7-8 are new to the Japanese school system, whereas children of 9 already have some experience.

\subsection{Robot Platform}

We used Robovie as robotic platform in the experiment (see Figures 1-4). It is a humanoid robot, designed for interaction with humans. It is $120 \mathrm{~cm}$ tall and $40 \mathrm{~cm}$ wide, weighing around $40 \mathrm{~kg}$. Robovie has two arms (4 x 2 DOF), a head with cameras that mimic robotic eyes (3 DOF) and it rolls on a mobile platform consisting in two driving wheels and one free wheel.

The robot played utterances in the experiment, which were displayed from pre-recorded messages with robotic voice and which we controlled following the Wizard-of-Oz technique. The main experimenter controlled Robovie's movement and most utterances. A second experimenter, who was a Japanese speaker, assisted the main experimenter whenever an understanding of the language was necessary.

\subsection{Environment}

The interaction with the robot took place in a room ample enough for the robot to roam around. The room presented a sofa and two sets of desks opposed to each other (see Figure 2). A large stuffed snake toy was available for the participant on top of another table. In preliminary trials we observed that children tended to grab the snake and occasionally disturb the robot with it. The robot operator sat in an adjacent room and controlled the scene via two cameras discreetly placed on the ceiling.

\subsection{Procedure}

In the following subsections we explain the steps of the procedure. In 3.5.1 we refer to how participants were prepared before interacting with the robot; afterwards, participants were made accustomed to the robot and underwent a first, fake experiment, which we describe in 3.5.2. In 3.5.3 we explain the subsequent, real experiment. Participants interacted with the robot individually.

\subsubsection{Preparation}

Prior to the experiment, the recruiting company debriefed the parents of the participants about the experiment and asked them to sign a consent form. Later on, the participant and a parent would arrive to our home institution, where they would be picked up by one of our experimenters. Subsequently, the participant was accompanied by the experimenter into the room adapted for the experiment.

\subsubsection{Habituation and fake experiment}

We gave great importance to the way how the robot was introduced to the participant. For example, the first interactions with the robot were dedicated to make the child feel more relaxed with the robot and minimize a possible initial anxiety. Once in the lab room, Robovie welcomed the participant and an experimenter encouraged him/her to touch the robot and talk to it.

After that, the participant was told that the experiment had already finished and was invited to sit on the sofa and answer a few questions about the robot (we are referring to this as the fake interview). Here, we told them that the experiment had already finished and asked them to wait in the room with the robot until their parents picked them up, consisting this waiting period in the actual experiment. This way, as we explained before, we expected to elicit more spontaneous and natural responses from the children.

\subsubsection{Main experiment}

Now that the fake experiment had concluded, we expected children to believe that they were now free and just waiting to be picked up by their parents. On the other hand, already since the participant moved to sit on the sofa for the fake interview, the robot had been roaming the room, carrying boxes from one side to the other, following a trajectory as illustrated in Figure 2. We designed this task so that the robot appeared to be doing something, giving the participant the possibility of interrupting it. 


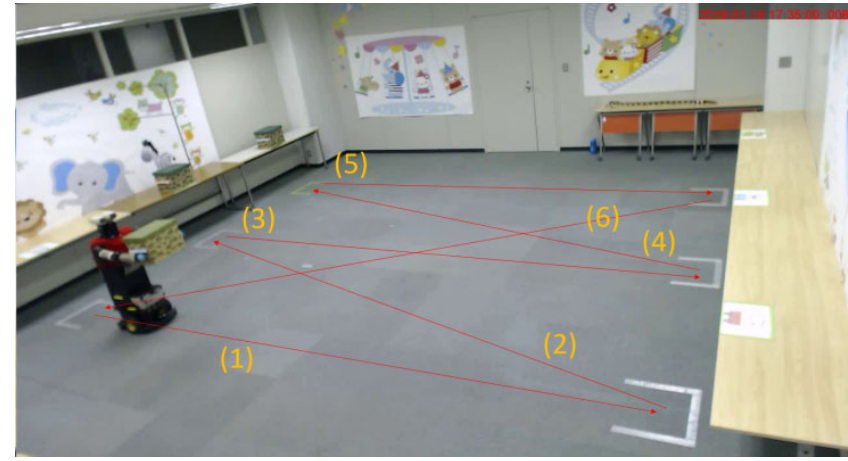

Figure 2: Trajectory the robot follows when moving boxes from one side of the room to the other.

Immediately after the fake interview, the experimenter explained that she needed to go somewhere else, asking the participant to remain alone in the lab room, with the robot, until he/she was picked up again. Thus, besides waiting, the participant had no specific instructions about how to behave toward the robot. Before leaving, the experimenter walked in front of the robot, obstructing its path. At that point, the robot would say "Excuse me, I would like to pass through". The experimenter would then apologize and the robot would say "thank you!". This showed the participant that the robot "does not like" to have its path blocked while performing its box moving task, serving as an example of task interruption.

Once the participant was left alone with the robot, if he/she interrupted the robot's task, this displayed utterances that corresponded to a given discipline strategy (more about this below). After 30 minutes of interaction time with the robot (or 20 minutes if the participant did not interact at all, for example if he/she had fallen asleep, in which case we decided sooner that they would be discarded for analysis), the experimenter came back, explained that this time alone with the robot was the actual experiment and conducted then the real interview.

Since we intended for the participants' behaviors toward the robot to be as spontaneous as possible, the robot did not initiate any interaction with them, being its behavior limited to moving the boxes. The robot also did not react initially to children's behaviors that were not considered disturbing for the robot and its task.

\section{Interruption of robot's task:}

When the child disturbed the robot or performed a behavior that interrupted its task, this displayed utterances that, depending on condition, conveyed the discipline strategy.

The robot operator deemed a child behavior as belonging to this type if such behavior obstructed the robot's performance (e.g. by blocking its path) and if there was an apparent intentionality to disturb. For example, sometimes a participant would stand so close to the robot that, due to the anti-collision system, its proximity sensors would prevent it from moving. If the participant unwillingly occasioned this, while giving enough room to the robot to move frontally, that would behavior would not be considered a disruptive behavior. The opposite would be a situation in which a participant decisively jumps on the path of the robot and shows unwillingness to let the robot move forward.
Other frequent examples of children's behaviors considered to disturb the robot were moving a box away when the robot was about to grasp it, putting the snake toy on top of the robot and altering the boxes in some way (e.g. removing a box lid), etc.

\subsection{Experimental Conditions}

We applied two experimental conditions, one in which the robot acted according to love-withdrawal strategies and bonding techniques; and another in which the robot's behaviors did not follow any specific discipline or persuasion strategy, which we call neutral condition. The experiment corresponds to a between-participants design. Table 1 offers an overview of the differences in the robot's behavior between the love-withdrawal and the neutral conditions.

\section{Love-withdrawal condition:}

This condition consists in behaviors that follow a lovewithdrawal strategy (e.g. utterances asking the participant to comply), as well as robot bonding behaviors that serve to strengthen the bond between child and robot, necessary for a love-withdrawal strategy to work. In order for the robot to bond with the child more than in the neutral condition, its behaviors have been made more empathic and interactive.

\begin{tabular}{|c|c|c|}
\hline Element & $\begin{array}{l}\text { Neutral } \\
\text { condition }\end{array}$ & $\begin{array}{l}\text { Love-withdrawal } \\
\text { condition }\end{array}$ \\
\hline $\begin{array}{l}\text { Compliance } \\
\text { utterance }\end{array}$ & $\begin{array}{l}\text { Neutral } \\
\text { message }\end{array}$ & Love-withdrawal message \\
\hline \multicolumn{3}{|l|}{ Thanking message: } \\
\hline Praise & No & Yes \\
\hline Name of participant & No & Yes \\
\hline Speech (only once) & $\begin{array}{l}\text { Neutral } \\
\text { content }\end{array}$ & $\begin{array}{l}\text {-Disclosure of robot's emotions } \\
\text {-Disclosure of secret } \\
\text {-Asks child to be its friend }\end{array}$ \\
\hline $\begin{array}{l}\text { Reacts to general } \\
\text { questions }\end{array}$ & No & Yes \\
\hline $\begin{array}{l}\text { Answer to playing } \\
\text { together }\end{array}$ & $\begin{array}{l}\text { Neutral } \\
\text { message }\end{array}$ & Love-withdrawal message \\
\hline Looks at participant & No & Yes \\
\hline
\end{tabular}

Table 1: Specific behaviors for Neutral and Lovewithdrawal conditions.

After the participant's first disruptive behavior, the robot displayed a compliance utterance asking the participant to stop it. For instance, the compliance utterances included "If you don't move out of the way, it will make me feel sad" ("path obstruction", i.e. situations in which the participant blocked the way of the robot), "If you don't stop that, I will be annoyed" ("general misbehavior", i.e. other misbehaviors besides path obstruction), etc. Once the participant stopped, the robot thanked him/her for stopping. An example of thanking utterances is "Thank you, (name of child)-chan, you are so kind", in which the name of the participant with a diminutive is included and there is praising. 
Subsequently, the robot turned toward the participant and emitted a short speech. This was included to strengthen the bond with the child. In the speech, the robot spoke about itself and told the secret (self-disclosure) that it felt a little lonely in the lab. It added that it liked the participant and also proposed him/her to be friends. After the participant answered, the robot resumed its task.

If the child disturbed the robot again, the robot emitted a compliance utterance and after he/she had complied (stopped the disruptive behavior), the robot used a thanking utterance. This sequence (child's disruption; robot's compliance utterance; child's stopping of behavior; robot's thanking utterance) was repeated indefinitely until the end of the interaction. When the participant asked the robot a question, the robot could reply with a short general answer that the robot operator selected from a pool of potential improvised answers. Examples are "yes", "no", "I don't understand", "that is great!", etc. If the participant asked the robot explicitly to play or do something together, this would reply with a sentence similar to "I'm sorry, I'm busy, but you can observe me nearby if you want", thus inviting for interaction while keeping busy on the task. Finally, in this condition, after the first disruptive behavior the robot would periodically briefly turn its body toward the child as if looking at him/her, which we expected to contribute to the bonding.

\section{Neutral condition:}

This condition followed the same general pattern as the lovewithdrawal condition, but the robot's utterances did not follow a specific discipline strategy. Thus, the robot displayed a compliance utterance asking the participant to stop his/her disruptive behaviors whenever such were produced (e.g. "please, stop doing that" for general behaviors, and "please, let me pass through" for path obstruction). When the participant stopped those behaviors, the robot thanked the participant (e.g. "thank you, I appreciate it". The short speech that came after the first disruptive behavior intended to be neutral in content, with the robot speaking about the lab and its robotic components and asking where the participant lives. After the participant answered, the robot resumed its task. To avoid repetitiveness, several utterances had been made for each robot message.

\subsection{Data Collection and Measures}

We aimed to measure the observable behavior of participants toward the robot. To that end, behavioral data were collected from two cameras discreetly located on the ceiling of the lab room and an interview at the end of the interaction with the robot provided us with subjective data.

\subsubsection{Behavior}

For each participant we counted the number of times that this initiated any disruptive behavior toward the robot (see subsection 3.5.3), whereby the robot would react, as well as the type of behavior. Regarding the latter, from a preliminary experiment we had learned that the main specific disruptive behaviors of children could be clustered into three categories, namely:

blocking the robot's path or preventing the robot from advancing and continuing with its task (for example, by standing in front of the robot so that this could not move forward, or putting something on the floor in the robot's trajectory, see Figure 1);

- manipulating the boxes in a disruptive way, for example, removing from the table the box that the robot is about to grasp (see Figure 3);

- and disturbing the robot with the snake toy, for instance by hanging it on the robot (see Figure 4).

Thus, we considered as dependent variables the total amount of disruptive behaviors toward the robot, as well as the aforementioned three categories.

We had noticed in preliminary tests that, regardless of any experimental treatment, some children tended to disturb the robot more than others. Thus, to help us separate these individual differences of the participants from the effectiveness of the experimental conditions, we considered introducing a concomitant variable. Namely, we measured the time it took participants to disturb the robot for the first time, that is, before the robot had started to behave differently depending on condition. From now on we will refer to this variable as "individual differences".

\subsubsection{Interview}

Participants were asked a few questions to ensure they had understood the robot's utterances. Next, they were asked why they had disturbed the robot. If they repetitively disturbed the robot, they were also asked why they disregarded the robot's requests to stop such behaviors. Likewise, they were asked why they stopped the disruptive behaviors if these had occurred just once (why tried to ascertain if this was due to the robot's requests). Finally, to establish differences between the two conditions in terms of bonding, children were asked whether they thought the robot was their friend and if they were going to miss the robot.

\section{RESULTS}

Before delving into the details, it may be worth giving a general description of the child-robot interactions that took place. Noticeably, children varied greatly in terms of how they interacted with the robot, not only in the amount of disruptive behaviors, but also in the type of behavior (e.g. path obstruction, or manipulating the boxes or the toy snake). Their behaviors also varied regarding time patterns: some children disturbed the robot especially at the beginning and then they would stop for the rest of the experiment, whereas other children showed a pattern of behaviors more spread in time. Another relevant observation we made was that children seemed to combine the disruptive behaviors with curiosity and collaboration. For instance, it was common to observe how the same child that disturbed the robot on numerous occasions also tried to "help" it (e.g. when grasping a box). Also, it may be worth reporting that, most of the times, children stopped their disruptive behaviors immediately as soon as the robot asked it. If they were to disturb the robot again, they would let some time pass first and then, iteratively, keep disturbing the robot again and again.

We will now show a description of the data, followed by ANCOVA analyses and finally the results from the interview analyses. Throughout our analyses, we considered the variables in Section 3.7.1 as our dependent variables. Even though our main independent variable of interest was Condition (with levels 
"love-withdrawal" and "neutral"), we also explored the influence of other available variables such as Gender and Age of the participants.

\section{Descriptive statistics:}

Prior to the main statistical analyses, we will report descriptive statistics for a better understanding of the data.Participants disturbed the robot or interrupted its activity around 7 times $($ Mean $=7.61, \mathrm{SD}=7.89)$, with a total of 464 disruptive actions. Per condition: love-withdrawal (Mean $=6.44, \mathrm{SD}=5.78)$; neutral $($ Mean $=8.90, \mathrm{SD}=9.64)$. The average number of these behaviors was 8.56 for the boys and 5.65 for the girls. The following is the decomposition of the disruptive behaviors into its main variants: blocking the robot's path (see Figure 1$)$, $($ Mean $=3.39, \mathrm{SD}=3.30)$; manipulating boxes in a disruptive way, $($ Mean $=1.92, \mathrm{SD}=$ $2.98)$; using the snake toy to disturb the robot, $($ Mean $=1.98, \mathrm{SD}$ = 3.12). We also measured the individual differences as defined in Section 3.7.1, $($ Mean $=247.26 \mathrm{~s}, \mathrm{SD}=285.86 \mathrm{~s})$.

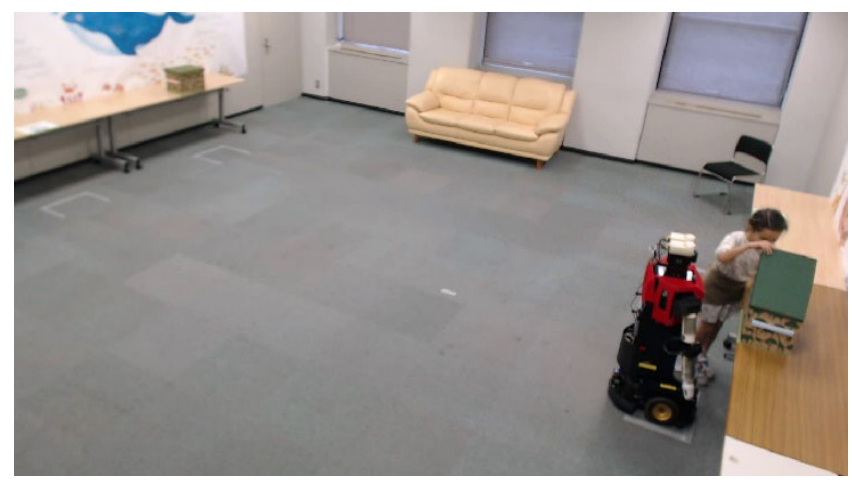

Figure 3: A participant manipulating a box when the robot was about to grasp it, thus interrupting.

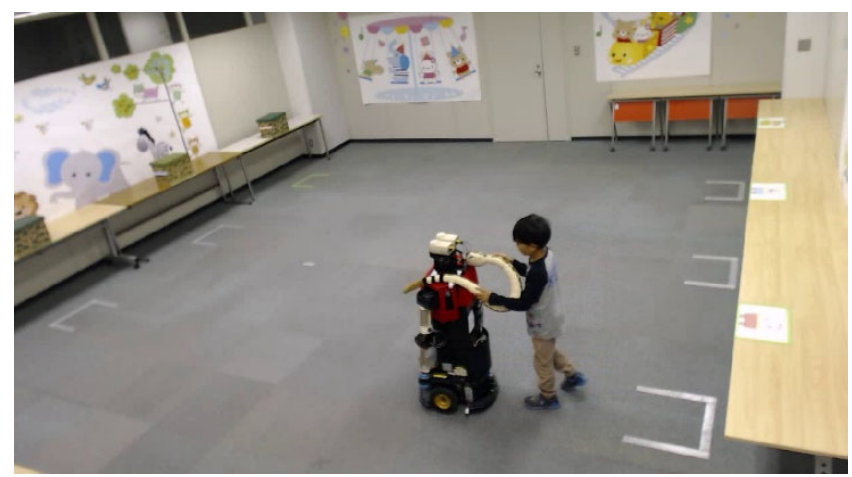

Figure 4: A child hanging the stuffed snake toy on the robot.

\section{Results from ANCOVAs:}

We conducted ANCOVAs for each of the dependent variables we previously described, having as independent variables Condition ("love-withdrawal" or "neutral"), Gender ("male" or "female") and Age (7-8 and 9). We kept the individual differences variable as a covariate in these tests.

In the ANCOVA that included general disruptive behaviors we did not find significant main effects for Condition $(\mathrm{F}(1,52)=$ 2.007, $\mathrm{p}=.162)$, Age $(\mathrm{F}(1,52)=0.680, \mathrm{p}=.413)$ and Gender $(\mathrm{F}(1$,
$52)=1.728, p=.194)$, but we found a significant effect of the covariate variable Individual Differences, $\mathrm{F}(1,52)=18.605, \mathrm{p}<$ .000 . We also found a trend in the interaction between Condition and Gender (female, love-withdrawal: Mean $=8.03, \mathrm{SD}=8.05$; female, neutral: Mean $=3.94, \mathrm{SD}=9.38$; male, love-withdrawal: Mean $=6.64, \mathrm{SD}=7.98$; male, neutral: Mean $=8.68, \mathrm{SD}=8.15$ ), $\mathrm{F}(1,52)=3.276, \mathrm{p}=.076$. Given that thus far we did not find significant effects involving Condition, we proceeded to analyze the three variants of the disruptive behaviors separately.

When we ran the ANCOVA with the disruptive behaviors related to path blocking as dependent variable, we did not find significant main effects for variables Condition $((\mathrm{F}(1,52)=0.929$, $\mathrm{p}=.340)$, Age $(\mathrm{F}(1,52)=0.088, \mathrm{p}=.768)$, nor Gender $(\mathrm{F}(1,52)=$ $0.001, p=.976)$. However, we again found a significant effect of covariate Individual Differences $(\mathrm{F}(1,52)=20.922, \mathrm{p}<.000)$. We also found a significant effect of the interaction between Condition and Age (Figure 5), F(1, 52) = 6.265, p = .016. Tukey's HSD post hoc tests maintained a trend when comparing "Lovewithdrawal" $($ Mean $=4.67, \mathrm{SD}=2.93)$ and "Neutral" $($ Mean $=$ $1.82, \mathrm{SD}=2.30)$ in $7-8$ years old participants $(\mathrm{p}=.058)$. Namely, the number of path blockings was higher in the love-withdrawal condition than in the neutral condition. However, in 9 years old participants, the contrast was clearly not significant $(\mathrm{p}=.320)$.

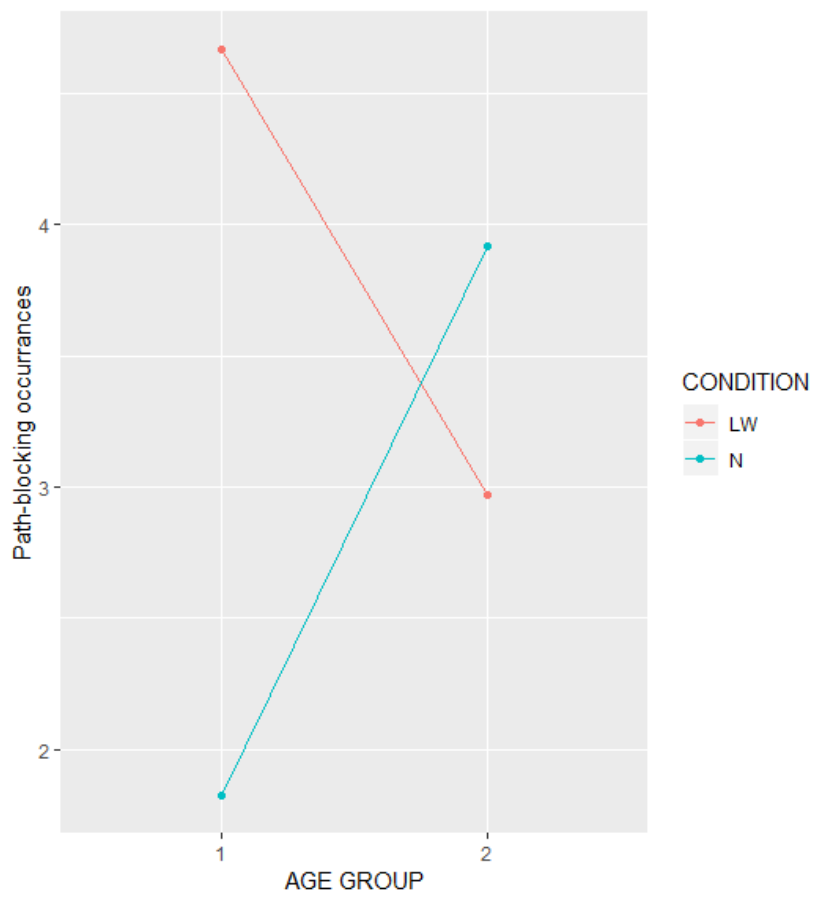

Figure 5: Statistical interaction between Age (7-8 vs. 9 years old) and Condition (love-withdrawal vs. neutral)

The ANCOVA that targeted disruptive behaviors involving use of the boxes showed no significant effects for Condition $(\mathrm{F}(1,52)$ $=1.887, \mathrm{p}=.175)$ nor Age $(\mathrm{F}(1,52)=1.964, \mathrm{p}=.167)$. We found $\mathrm{a}$ trend in the main effect of Gender, $(\mathrm{F}(1,52)=3.730, \mathrm{p}=.059)$. As for the interaction between Condition and Gender, we did not find a significant effect, $F(1,52)=2.054, p=.158$. Also, we found again a significant effect of covariate Individual Differences $(\mathrm{F}(1$, 52) $=9.827, \mathrm{p}=.003)$. 
Finally, the ANCOVA that considered disruptive behaviors related to the toy snake did not show significant results for Condition $(\mathrm{F}(1,52)=1.477, \mathrm{p}=.230)$, Age $(\mathrm{F}(1,52)=0.125, \mathrm{p}=$ $.725)$, nor Gender $(\mathrm{F}(1,52)=2.281, \mathrm{p}=.137)$. We found a significant effect of the Individual Differences covariate, $\mathrm{F}(1,52)$ $=4.842, \mathrm{p}=.032$.

We did not apply Bonferroni correction for the analysis above given that the three variants of disruptive behavior consisted in three different dependent variables.

\section{Analysis of interview answers:}

As reaction to the question "why did you disturb the robot?" participants tended to give the same answers, namely, "because they wanted to see the robot's reaction" (29 participants), "because they were bored" (6 participants), or "because they wanted to talk with the robot" (11 participants). 13 participants had other various reasons and 2 were not applicable (see Table 2 for a counting per condition). A Chi-squared test showed a statistical trend between the two conditions as predictors of the abovementioned categories of answers $\left(\chi^{2}=7.10, \mathrm{df}=3, \mathrm{p}=.07\right)$.

\begin{tabular}{|l|l|l|l|l|}
\hline CONDITION & $\begin{array}{l}\text { See } \\
\text { reaction }\end{array}$ & Bored & $\begin{array}{l}\text { Talk to } \\
\text { robot }\end{array}$ & Others \\
\hline $\begin{array}{l}\text { Love- } \\
\text { withdrawal }\end{array}$ & 14 & 1 & 9 & 7 \\
\hline Neutral & 15 & 5 & 2 & 6 \\
\hline
\end{tabular}

Table 2: counting of answers to question "Why did you disturb the robot?"

\section{DISCUSSION}

\subsection{Summary and Implications}

While we acknowledge the great differences between this and previous studies on robot abuse by children ([11], [12]), we found some similarities in terms of the behaviors shown by children. For instance, blocking the robot's path also appears to be the most common disruptive behavior in the previous studies. However, as we explained, we took a further step into preventing children from abusing robots.

With respect to the observable measures, we found a significant effect of the individual differences in the disruptive behaviors as a whole as well as in all its subcomponents. We will discuss this finding later.

Also considering the disruptive behaviors as a whole, although still a trend, we found indications of a possible interaction between Condition and Gender. The love-withdrawal condition would be more effective than the neutral condition in the case of the male participants, but in the case of female participants the neutral condition may be more effective. A conjecture to explain this might come from general differences between boys and girls of that age. We could argue that boys tend to be more proactive and bolder (they also tended to disturb the robot more often). Thus, the love-withdrawal intervention might work better with boys because they represent better the kind of target participant we had in mind when we designed the experiment. On the other hand, Japanese girls of this age tend to be more passive. When they found a Robovie that was less interactive and friendly in the neutral condition, they may have gone along with the robot's behavior and interacted less with it (which included less disruptive behaviors).

Besides this, the only clear statistically significant difference was found in the interaction between Age and Condition, as predictors of the path-blocking disruption variant. More precisely, the neutral treatment seemed most effective with the 7-8 years old children, whereas the love-withdrawal treatment appeared more effective with the 9 years old children. In other words, the love-withdrawal condition seemed more effective only with the older children, whereas with 9 years old children its effect seems even detrimental. This finding points at the importance of considering the exact age of children when we design a Child-Robot Interaction intervention. Indeed, children around the ages of 7 to 9 change not just physically, but also cognitively, at a quite rapid pace [17].

Continuing with the influence of gender, we also found in a trend that the girls seemed to disturb the robot less often by manipulating the boxes, as opposed to the boys. This appears congruent with the finding that girls tended to disturb the robot overall less often than boys, as we have seen before. The fact that girls at this age show less abuse than boys does not seem to be a counterintuitive fact.

With respect to the data analysis from the interview answers, again we only obtained a statistical trend when exploring the differences between conditions. However, a close look at Table 2 may reveal some aspects in which the two conditions may have worked in the way they were intended to. Namely, in the neutral condition there were more children that disturbed the robot out of boredom (thus with the effect that we intended, since Robovie was less interactive and more "selfish" in the neutral condition). On the other hand, there were more children in the lovewithdrawal condition than in the neutral condition who disturbed the robot because they wanted to talk to it (thus in line with a robot that is more interactive and "mindful" about the child's presence). Children might have disturbed the robot because it attracted their interest more.

\subsection{Discipline Styles}

Now, given these results, do we have enough evidence to believe that a love-withdrawal intervention would lower the amount of disruptive behaviors towards a robot? We learned that the effectiveness of such intervention seems to be greatly dependable on several factors, such as gender and age. Also, we found that individual differences of children (related to their proneness to start disturbing the robot) were a reliable predictor of robot abuse. What this seems to suggest is that that a "onesize-fits-all" perspective on robot behavior might be inadequate in this kind of situation. Perhaps we should start by identifying what robot behaviors and personalities are more suitable to what types of children. For instance, in this study we learned that a robot using a love-withdrawal strategy might not be effective on younger children.

A major limitation of the present study lies in the adaptation of the love-withdrawal strategy to an HRI context. Hoffman's discipline strategies [18] take place in the highly complex environment of a family and over extended periods of time. While we acknowledge the irreproducibility of this kind of 
parental strategy under our lab conditions, at least in the strict sense, we incorporated some of its elements in our experiment. Namely, in the love-withdrawal condition we aimed to strengthen the short-term bond that could arise between the child and the robot; and when the child misbehaved the robot emitted "threats" of breaking the bond if the child did not comply.

Another limitation in the present study may lie in the definition of robot abuse. For simplicity, we have identified "robot abuse" with "disruptive behaviors" directed at the robot when this is performing a task. Some may argue that such identification is inadequate and that indeed insulting or physically attacking the robot (as in [11] , [12]) are children behaviors quite different in nature to the behaviors we have encountered here. Such comment may be legit. In any case, we have worked with the kind of children behaviors that we spontaneously found in the lab.

Finally, another limitation may refer to the combination we used of multiple robot bonding techniques together with the condition-specific robot behaviors (e.g. love-withdrawal strategy in the love-withdrawal condition). This makes it more difficult to draw conclusion about what exactly caused the effects associated to the two conditions.

To conclude, we did not debrief the children after the experiment regarding the fact that the robot had been operated through Wizard-of-Oz. Although our study was IRB-approved, we would like to note that in future studies we would like to follow the good practice of debriefing children about this fact, so as to avoid creating false beliefs about the robot capabilities.

\section{CONCLUSION}

We designed a Human-Robot Interaction intervention aiming to reduce the occurrence of robot abuse by children. We applied parental discipline strategies as described by Hoffmann, namely the love-withdrawal discipline style [18], to the set of behaviors of the robot, and we compared this strategy to a set of robotic behaviors that were not based on any specific strategy (neutral condition). From the results, it seems that there might be no single strategy to fit all types of children. Rather, it appears that different strategies might have to be applied depending on the characteristics of the children. For instance, we found that the love-withdrawal strategy might work better with children 9 years old than with children 7-8 years old; and we also found indications that the same strategy might be more effective on boys than on girls.

In the present study, children interacted individually with the robot. However, the most aggressive forms of robot abuse by children which we find in the literature seem to take place when children are in groups ([11], [12]). Thus, something we may want to consider for future work is having groups of children (e.g dyads) interacting with a robot in the lab.

Another possibility for future work regards the types of strategies adapted for robot behavior. In the present study, we only considered Hoffmann's love-withdrawal discipline strategy. However, we could also explore Hoffmann's induction or power assertion discipline strategies, or even other strategies from other authors. In any case, we have to sum the present study to previous ones (e.g. [11], [12]) which already pointed out how difficult it is to control the abuse that children sometimes exert on robots. This matter, therefore, still remains as an open challenge to embrace in the HRI field.

\section{ACKNOWLEDGMENTS}

This research was supported by JST CREST Grant Number JPMJCR17A2, Japan. We would also like to thank our assistants for their work.

\section{REFERENCES}

[1] S. Turkle, C. Breazeal, O. Dasté, and B. Scassellati, "Encounters with kismet and cog: Children respond to relational artifacts," Digit. Media Transform. Hum. Commun., 2006.

[2] C. P. Flynn, "Animal Abuse in Childhood and Later Support for Interpersonal Violence in Families," Soc. Anim., 1999.

[3] R. Sparrow, "Robots, Rape, and Representation," Int. f. Soc. Robot., vol. 9, no. 4, pp. 465-477, 2017.

[4] B. Whitby, "Sometimes it's hard to be a robot: A call for action on the ethics of abusing artificial agents," Interact. Comput., vol. 20, no. 3, pp. 326-333, 2008.

[5] R. B. Patrick and J. C. Gibbs, "Parental expression of disappointment: Should it be a factor in Hoffman's model of parental discipline?," 7 . Genet. Psychol., vol. 168, no. 2, pp. 131-145, 2007.

[6] T. Belpaeme et al., "Child-robot interaction: Perspectives and challenges," in Lecture Notes in Computer Science (including subseries Lecture Notes in Artificial Intelligence and Lecture Notes in Bioinformatics), 2013.

[7] T. Kanda, T. Hirano, D. Eaton, and H. Ishiguro, "Interactive Robots as Social Partners and Peer Tutors for Children: A Field Trial," HumanComputer Interact., vol. 19, no. 1, pp. 61-84, 2004.

[8] F. Tanaka and S. Matsuzoe, "Children Teach a Care-Receiving Robot to Promote Their Learning: Field Experiments in a Classroom for Vocabulary Learning," 7. Human-Robot Interact., 2012.

[9] E. Short et al., "How to train your DragonBot: Socially assistive robots for teaching children about nutrition through play," in IEEE RO-MAN 2014 - 23rd IEEE International Symposium on Robot and Human Interactive Communication: Human-Robot Co-Existence: Adaptive Interfaces and Systems for Daily Life, Therapy, Assistance and Socially Engaging Interactions, 2014.

[10] B. Robins, K. Dautenhahn, R. Boekhorst, and A. Billard, "Effects of repeated exposure to a humanoid robot on children with autism," in Cambridge Workshop Universal Access and Assistive Technology (CWUAAT), 2004, no. March, pp. 225-236.

[11] P. Salvini et al., "How safe are service robots in urban environments? Bullying a robot," 19th Int. Symp. Robot Hum. Interact. Commun., pp. 17, 2010 .

[12] D. Brscić, H. Kidokoro, Y. Suehiro, and T. Kanda, "Escaping from Children's Abuse of Social Robots," in Proceedings of the Tenth Annual ACM/IEEE International Conference on Human-Robot Interaction - HRI '15, 2015.

[13] T. Kanda, M. Shiomi, Z. Miyashita, H. Ishiguro, and N. Hagita, "An affective guide robot in a shopping mall," Proc. 4th ACM/IEEE Int. Conf. Hum. Robot Interact. - HRI '09, p. 173, 2009.

[14] A. Waytz, C. K. Morewedge, N. Epley, G. Monteleone, J. H. Gao, and J. T. Cacioppo, "Making sense by making sentient: effectance motivation increases anthropomorphism.," f. Pers. Soc. Psychol., vol. 99, no. 3, pp. 410-435, 2010.

[15] J. Fasola and M. J. Matarić, "Using Socially Assistive Human-Robot Interaction to Motivate Physical Exercise for Older Adults," Househ Serv. Robot., vol. 100, no. 8, pp. 487-516, 2014.

[16] C. D. Kidd and C. Breazeal, "Robots at home: Understanding long-term human-robot interaction," 2008 IEEE/RSf Int. Conf. Intell. Robot. Syst., pp. 3230-3235, Sep. 2008.

[17] J. Piaget, "Part I: Cognitive development in children: Piaget. Development and learning," f. Res. Sci. Teach., vol. 2, no. 3, pp. 176186, 1964.

[18] M. L. Hoffman, "Empathic Anger, Sympathy, Guilt, Feeling of Injustice," Empathy moral Dev. Implic. caring justice, 2000. 